\title{
Surgical Approaches to Resection of Anterior Skull Base and Paranasal Sinuses Tumors
}

\author{
Sara Abu-Ghanem, Dan M. Fliss \\ Department of Otolaryngology Head and Neck Surgery and Maxillofacial Surgery, Tel-Aviv Sourasky Medical Center, Sackler School \\ of Medicine, Tel Aviv University, Tel Aviv, Israel
}

\begin{abstract}
Malignant tumours of the sinonasal tract comprise approximately $3 \%$ of the malignancies that arise in the upper aerodigestive tract. Approximately $10 \%$ of tumours that arise in the sinonasal tract originate in the ethmoid and/or frontal sinuses, and are likely to involve the anterior cranial base. The route of spread of tumours originating in the anterior skull base and paranasal sinuses is determined by the complex anatomy of the craniomaxillofacial compartments. These tumours may invade laterally into the orbit and middle fossa, inferiorly into the maxillary antrum and palate, posteriorly into the nasopharynx and pterygopalatine fossa, and superiorly into the cavernous sinus and brain. Recent improvements in endoscopic technology now allow the resection of the majority of benign neoplasms and some early malignant tumours with minor dural involvement. For advanced-stage malignant tumours and benign tumours with frontal bone involvement, the classical open approaches remain viable surgical techniques. In this paper, we review the open surgical resection approaches used for resections in the craniomaxillofacial area.
\end{abstract}

Key Words: Anterior skull base, open approaches, paranasal tumours

Received: 11.03.2013

Accepted: 19.03.2013

\section{Introduction}

Tumours involving the anterior skull base and paranasal sinuses are challenging to treat because of their relative rarity, the wide diversity of tumour types represented, and the variability in the extent of involved local structures (1).

The route of spread of these tumours is determined by the complex anatomy of the craniomaxillofacial compartments. These tumours may invade laterally into the orbit and middle fossa, inferiorly into the maxillary antrum and palate, posteriorly into the nasopharynx and pterygopalatine fossa (PPF), and superiorly into the cavernous sinus and brain. Surgery remains the important modality of treatment of anterior skull base tumours. Combined craniofacial techniques for the resection of tumours of the anterior skull base were first described by Ketcham et al. (2) in 1963. Since then, anterior skull base surgery has evolved greatly, with a better understanding of the anatomy, pathology, imaging and surgical techniques. The craniofacial approach and the subcranial approach have become the standard of care for the treatment of malignant tumours involving the anterior skull base (3-6) and, because of this and the varied histologic findings, most outcomes data reflect the experience of small patient cohorts. This International Collaborative study examines a large cohort of patients accumulated from multiple institutions experienced in craniofacial surgery, with the aim of reporting benchmark figures for outcomes and identifying patient-related and tumor-related predictors of prognosis after craniofacial resection (CFR. Recent developments in technology have allowed the use of endoscopic endonasal approaches. Nevertheless, several problems still exist, relating to case selection, histology, cost, patient selection, and the experience of the surgeon. This review focuses on the open surgical resection approaches used for anterior skull base tumours, as used in our institution in the last 20 years.

\section{Anatomy}

The anterior skull base is a complex anatomical compartment. It can be defined as the portion of the skull base adjacent to the anterior cranial fossa. The anterior skull base boundaries include the medial border that is formed by the cribriform plate, making up the roof of the nasal cavity, the lateral border that is formed by the orbital plates of the frontal bone that form the roof of the orbits and ethmoid air cells, and the posterior border that is formed by the planum sphenoidale and lesser wings of the sphenoid (4).

The cribiform plate is traversed by multiple olfactory nerves that extend from the olfactory mucosa to the olfactory bulbs. A bony fissure between the lesser and greater sphenoid wings, the superior orbital fissure, gives passage to cranial nerves III, IV, V and VI and to the superior ophthalmic vein. Superior and laterally lies the optic canal bordered by the body of the sphenoid and by the superior and inferior roots of the lesser sphenoid wing, giving passage to the optic nerve, ophthalmic artery, and sympathetic nerves. However, both the superior orbital fissure and optic canal open in the middle cranial fossa and are most commonly affected by central skull base lesions (4). The thin cribiform plate is 
easily crossed by tumours; however, the orbital plates of the frontal bone are made of a thick compact bone that constitutes a barrier to tumour growth into the anterior cranial fossa. Therefore, it is not surprising that most tumours affecting the anterior skull base arise from the sinonasal region.

\section{Preoperative Evaluation and Anaesthesia}

All patients scheduled for operation are evaluated preoperatively by a head and neck surgeon, a neurosurgeon, an anaesthesiologist, and a reconstructive plastic surgeon. Patients younger than 18 years are also examined by a paediatrician. In the past decades, cross-sectional imaging has become a key factor in the management of skull base pathology. CT and MRI have a complementary role in the evaluation of skull base pathology and are often used together to determine the full extent of a lesion (4). In recent years, positron emission tomography (PET), often combined with $\mathrm{CT}$, has become popular. $\mathrm{PET} / \mathrm{CT}$ useful for determining the staging of the disease and identifying residual or recurrent disease $(5,7)$. Angiography is rarely used nowadays for diagnostic purposes. Highly vascular lesions, such as juvenile angiofibroma or paraganglioma, can be diagnosed with angiography. Angiography is also used for therapeutic purposes in the case of preoperative embolisation and balloon occlusion test.

\section{Open Surgical Approaches for the Anterior Skull Base}

Adequate exposure of the anterior cranial base for excision of neoplasms traditionally requires a combined intracranial and extracranial approach. Most commonly, a team of neurosurgeons and otolaryngologists performs this procedure. The choice of an extracranial approach depends on the site and extent of the tumour and aesthetic considerations, as well as the experience of the surgeon with particular approaches.

The extent of exposure of these approaches include the frontal sinus anteriorly, the clivus posteriorly, the frontal lobe superiorly, and the paranasal sinuses, the pterygo-maxillary fossa and infratemporal fossa inferiorly. The lateral boundaries of this approach include both superior orbital walls.

\section{Transfacial Approaches}

\section{Lateral Rhinotomy Incision}

The lateral rhinotomy approach is used in the case of a malignant tumour originating in the nasal cavity and maxillary sinus without palatal invasion. Benign tumours with anterior maxillary wall involvement are similarly approached. This approach allows wide exposure of the maxillary antrum, nasal cavity, ethmoidal sinuses, and sphenoid sinus. The facial incision extends along the lateral border of the nose, $\sim 1 \mathrm{~cm}$ lateral to the midline. It starts from the cephalad medial cantus and extends down through the skin crest bordering the nasal ala. It is continued towards the filtrum. The flaps can be developed to the level of the maxillary tuberosity laterally, the upper gingival sulcus inferiorly, the frontal sinus and infra-orbital rim superiorly, and to the nasion (suture between the frontal and nasal bones) and nasal septum medially.

\section{Weber-Fergusson Incision}

In cases of malignant tumours infiltrating the lateral maxillary wall or palate, total maxillectomy is performed via a Weber-Fergusson incision. This approach involves an extenuation of the lateral rhinotomy incision that includes splitting of the upper lip. The Weber-Fergusson incision permits complete exposure of the maxilla, from the upper alveolar ridge to the orbit. This allows exposure of the superior and inferior aspects of the maxilla and its complete en bloc resection. The soft tissue of the cheek is raised from the anterior surface of the maxilla, transecting the infra-orbital nerves and vessels should the superior and lateral walls of the maxilla need to be approached. An upper cheek flap is developed laterally and superiorly up to the level of the inferior orbital rim and the maxillary tuberosity. Inferiorly it can reach the pterygomaxillary fossa.

\section{Lynch Incision}

The Lynch incision is rarely used nowadays as a sole approach but rather, it is used as an extension of the Weber-Fergusson incision. This incision extends along the lower border of the eyebrow or in a skin crest along the upper lid, allowing it to be concealed at the hair-skin junction. If the incision is made inside the eyebrow, it may leave a thick and noticeable scar, giving inferior cosmetic results. The incision is extended down, $\sim 0.5 \mathrm{~cm}$ medial to the medial cantus. It can be extended laterally up to the level of the lateral cantus, or inferiorly to be included in a lateral rhinotomy incision.

\section{Dieffenbach Incision and Its Modifications}

The Dieffenbach incision and its modified forms are used to approach tumours involving the infra-orbital rim and zygomatic root. It can be extended up to the level of the medial cantus, or inferiorly, to be included in a lateral rhinotomy incision. The classical Dieffenbach incision extends along the lower border of the eyelid, along a skin crest. The incision extends from the medial cantus to the lateral cantus. A later modification of this incision is the subcilliary incision, which is performed just below the cilia of the eyelid, or the midcilliary incision, performed halfway between the Dieffenbach and subcilliary incisions.

The superior border of the flap includes the infra-orbital rim and orbit; its inferior border is the anterior maxillary wall; laterally it is extended to expose the maxillary tuberosity and root of the zygoma; and medially it extends to the nasal bone. In elderly and previously irradiated patients, the redundant skin and subcutaneous tissue of the lower eyelid tend to swell as the incision may include the lymphatic drainage of this area. The skin is closed with a subcutaneous, continuous number 5.0 prolene stitch to prevent contraction of the thin skin in this area. This incision is also almost exclusively used in conjunction with other transfacial skin incisions.

\section{Midfacial Degloving (MFD)}

The MFD approach combines the sublabial incision used in external approaches to sinus surgery with the intranasal incision used in cosmetic rhinological surgery. The main ad- 


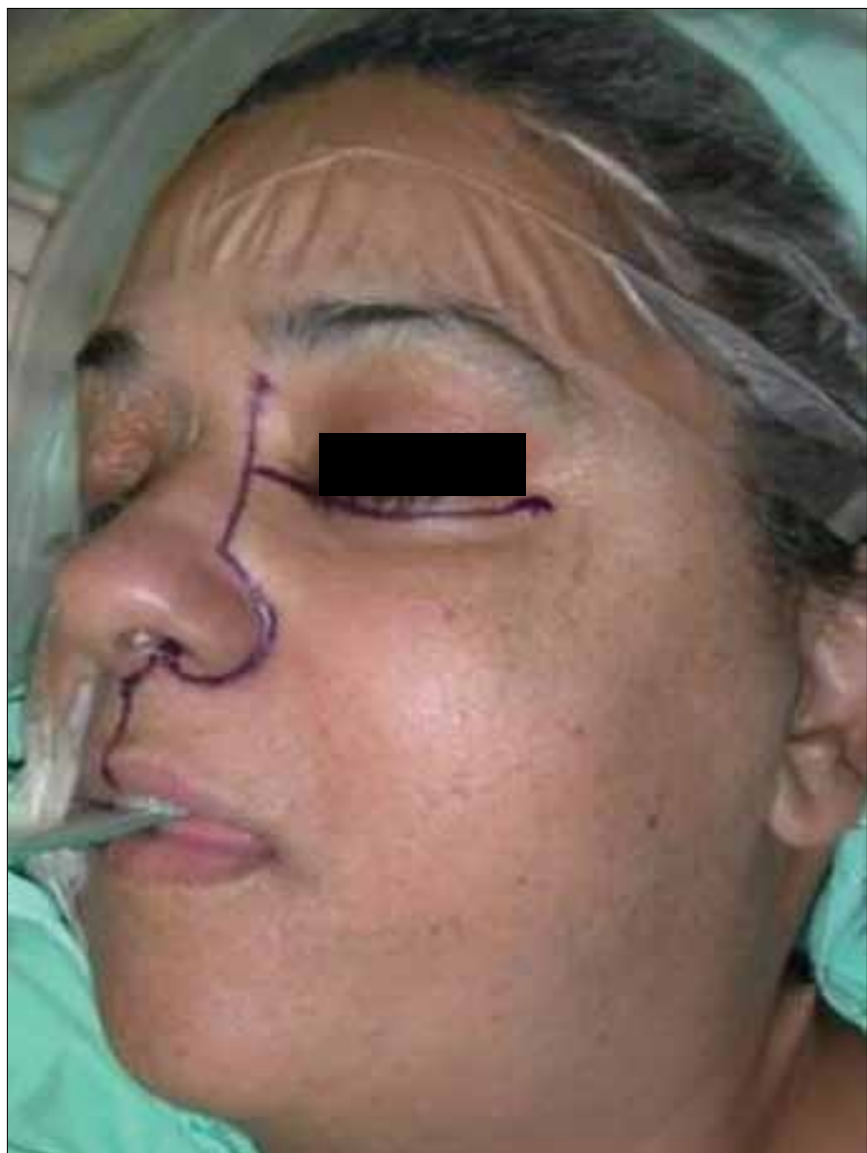

Figure 1. Facial incisions for the resection of craniofacial neoplasms: lateral rhinotomy, Weber-Ferguson, and midciliary. Combined approaches are possible

vantage of this approach over conventional lateral rhinotomy or Weber-Ferguson approaches is avoidance of facial incision. This approach was originally designed for benign tumours, including inverted papilloma, juvenile angiofibroma, odontogenic cysts, and benign fibro-osseous lesions (8). Nowadays, MFD has been largely replaced by the endonasal approach as the main technique for extirpation of these lesions. Nevertheless, it can be used for large tumours involving the anterior skull base, in conjunction with an 'upper' approach, or after the failure of an endonasal resection. The MFD approach involves a complete transfixation incision, with a complete intercartilaginous incision. This effectively separates the upper lateral cartilage from the lower lateral cartilage, the latter of which is later included with the superiorly retracted flap. Next, a 'degloving' of the facial soft tissue from the nasal skeleton and maxilla is preformed. This is achieved through a sublabial incision that extends from first molar to first molar. We have popularised the use of MFD in conjunction with the subcranial approach in order to restore the skull base tumour excision in its inferior-lateral and posterior extension (9). Figure 1 summarises the surgical transfacial approaches.

\section{Craniofacial Resection}

The craniofacial resection is a well-established technique for the surgical excision of tumours involving the anterior skull

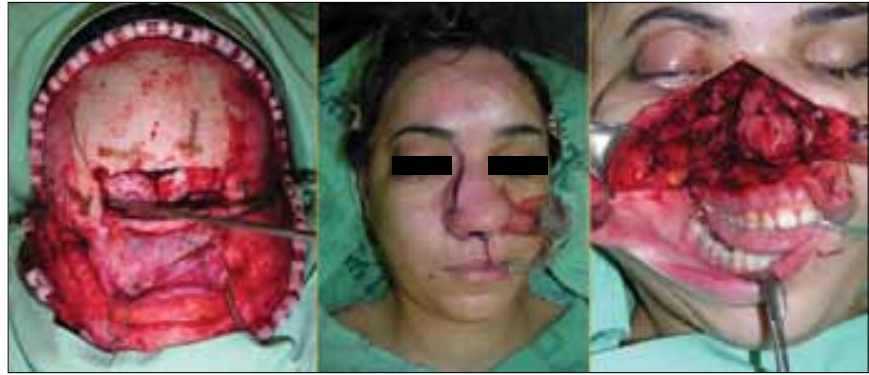

Figure 2. The craniofacial approach. A Weber-Fergusson incision (right) and coronal incision (left) are shown

base and paranasal sinuses $(3,10,11)$. This technique incorporates a combination of transfacial and transcranial procedures in order to allow broad exposure of the anterior cranial fossa and subcranial compartment. Initially, a lateral rhinotomy is made, followed by a medial maxillectomy, anterior and posterior ethmoidectomy and sphenoidectomy, which are preformed under a microscope. The cribriform plate and frontal recess are exposed along with the lamina papyracea bilaterally. Next, the elevation of the coronal flap and frontal craniotomy is performed. The craniotomy includes the frontal bone from the level of the glabela below, to roughly $4-5 \mathrm{~cm}$ above the skull base superiorly. The lateral borders of the craniotomy are the midpupillary line bilaterally. Next, the dura is incised, if indicated, and the frontal lobes are retracted superiorly, exposing the anterior skull base from above. The final stage of the operation includes resection of the tumour, which extends through the cribiform plate in a combined fation from below and above. The use of craniofacial surgery has been documented for a variety of malignant and benign tumours. This technique has two major advantages: (i) it affords broad exposure of the anterior skull base from above and below, providing excellent access to the orbital, spheno-ethmoidal and paranasal cavities; and (ii) resection of intradural and extradural tumours can be performed in a single procedure that allows precise reconstruction of the dura. Despite the technical reproducibility of the craniofacial approach, this procedure still involves a high risk of postoperative complication. The main limitation of this approach is the need for frontal lobe retraction, which may lead to encephalomalacia, brain oedema, and subdural bleeding, especially in the elderly population, and the presence of cosmetic inconvenience because of the transfacial skin incisions (Figure 2).

\section{The Subcranial Approach}

The subcranial approach is a single-stage procedure used for tumours involving the anterior skull base $(12,13)$. The extent of exposure with the subcranial approach includes the frontal sinus anteriorly, the clivus posteriorly, the frontal lobe superiorly and the paranasal sinuses inferiorly. Laterally, the boundaries of this approach are both superior orbital walls. The subcranial approach has several major advantages: (i) it affords direct exposure of the anterior skull base from anterior to posterior instead of from above and below, as in the craniofacial approach; (ii) it allows simultaneous intradural and extradural tumour removal from anterior to posterior; (iii) it does not require facial incision; and (iv) minimal frontal lobe manipulation is required. 


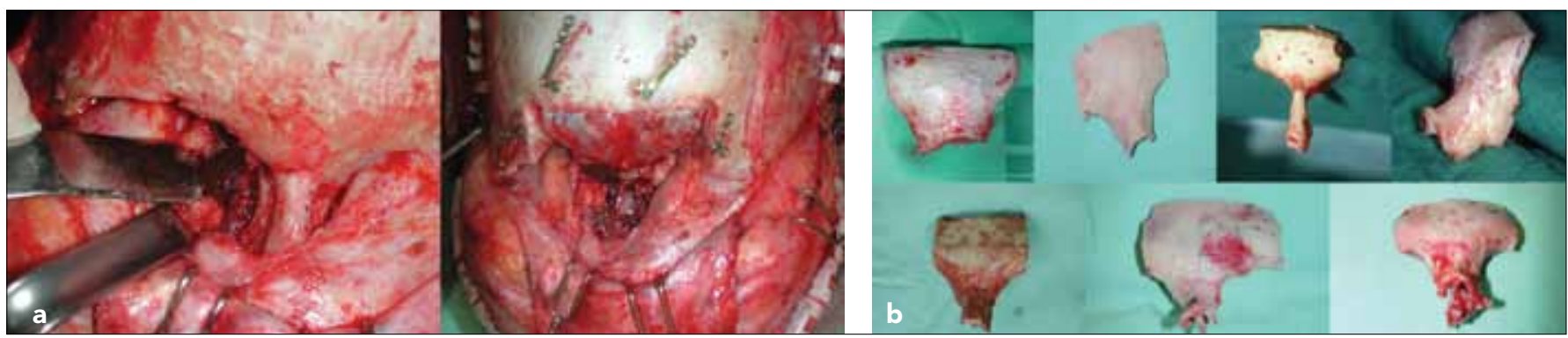

Figure 3. The subcranial approach. a) Intraoperative view showing the elevation of coronal and pericranial flaps and osteotomies (right). b) Nasofrontal bones after subcranial resection can be tailored according to the location and dimension of the anterior skull base

The subcranial approach involves a coronal incision and osteotomy of the naso-fronto-orbital bone segment, which allows access to the intra- and extra-cranial compartments of the anterior skull base (Figures $3 a$ and $3 b$ ). The main disadvantage of this approach is bone osteonecrosis post radiotherapy in cases of malignant tumours (14).

\section{The Subcranial Approach and Combinations}

Although both the subcranial and craniofacial approaches permit complete tumour resection in the majority of cases, situations still arise in which the inferior, lateral or posterior aspects of the tumour are not adequately exposed. These include neoplasms with extensions to the hard palate caudally, to the cavernous sinus posteriorly, to the orbital apex, PPF, or infratemporal fossa (ITF) laterally, and to the nasopharynx and clivus inferoposteriorly. Such cases require a combination of the standard subcranial approach with other approaches as a one-step procedure to allow proper exposure and tumour extirpation. In these combined approaches, a second approach may include one or more of the following procedures: MFD, orbito-zygomatic, transfacial, Le-Fort I, or the transorbital approach. These combined approaches require additional incisions and osteotomies, according to the type and extent of the tumour.

\section{In Combination with the Pterional Approach}

This approach is a combination of the subcranial approach and the pterional approach (15). A unilateral pterional approach is added to the subcranial approach to expose the orbit, the retro-orbital region, or the ITF. Large central nervous system tumours with extracranial extent, such as meningiomas involving the orbit or upper nasal and sinus cavities, can also be resected via this combined approach. In this modification, the coronal incision is preformed and the skin flap elevation continues down to the level of the fat pad overlying the zygoma, above the temporalis fascia. On the ipsilateral side, the reminder of the dissection dips below the level of the temporalis fascia from the horizontal line above the arch and continues as a fasicocutaneous flap. The muscle is detached anteriorly and superiorly, exposing the temporal fossa. The next stage includes osteotomies in both frontal and pterional regions. The standard osteotomy described above is modified by its extension laterally, to include a portion of the orbital roof and temporal bone. The bone segment is then removed in one or two pieces, exposing the orbital, ethmoid and sphe- noid roofs, the cribiform plate, the temporal fossa, and the parasellar area.

\section{In Combination with the MFD Approach}

The subcranial MFD approach is used for resecting benign tumours involving the anterior skull base and the inferior, lateral, and posterior planes of the maxillary sinus. This is indicated mainly for the resection of juvenile angiofibromas with anterior skull base invasion (9). It can be also combined with an orbitozygomatic or pterional approach for tumours that extend to the lateral skull base (Figure 4).

\section{In Combination with the Le Fort I Approach}

The subcranial-LeFort I approach allows a wide exposure of the tumour from the cribriform area to the lower part of the clivus, maxillary sinuses, and nasal cavity. It is indicated only for selected cases that cannot be approached by the subcranial approach or combined endoscopic-subcranial approach. This approach may be selected for the extirpation of large chordomas or chondrosarcomas originating in the clivus, which extend superiorly to the sphenoid sinus, planum sphenoidale, and cribriform plate.

\section{In Combination with the Transorbital Approach}

A combined transcranial-transorbital approach is used for malignant tumours that penetrate the bony orbit and periost and infiltrate the anterior orbital content (T4a) or orbital apex (T4b) (12). A coronal flap is performed and the flap is extended inferiorly in one side of the coronofacial flap (Figures $5 a$ and $5 b$ ). The superior and medial walls of the orbit are exposed and stripped from their periostium. During dissection along the medial orbital wall, the anterior and posterior ethmoidal arteries are identified and clipped. If the roof or medial orbital wall is involved by the tumour, they are removed at this stage. If they are not involved, the upper and lower lids may be spread, allowing future insertion of an orbital implant and an improved cosmetic result. If the lids are involved, a circular skin incision is made along the superior and inferior orbital rims and the skin of the lids is kept on the main specimen.

\section{Conclusion}

Anterior skull base surgery is a young discipline that originated only half a century ago. Detailed knowledge of skull base anatomy is a prerequisite for correct imaging diagnosis and for accurate delineation of the extent of skull base lesions. 


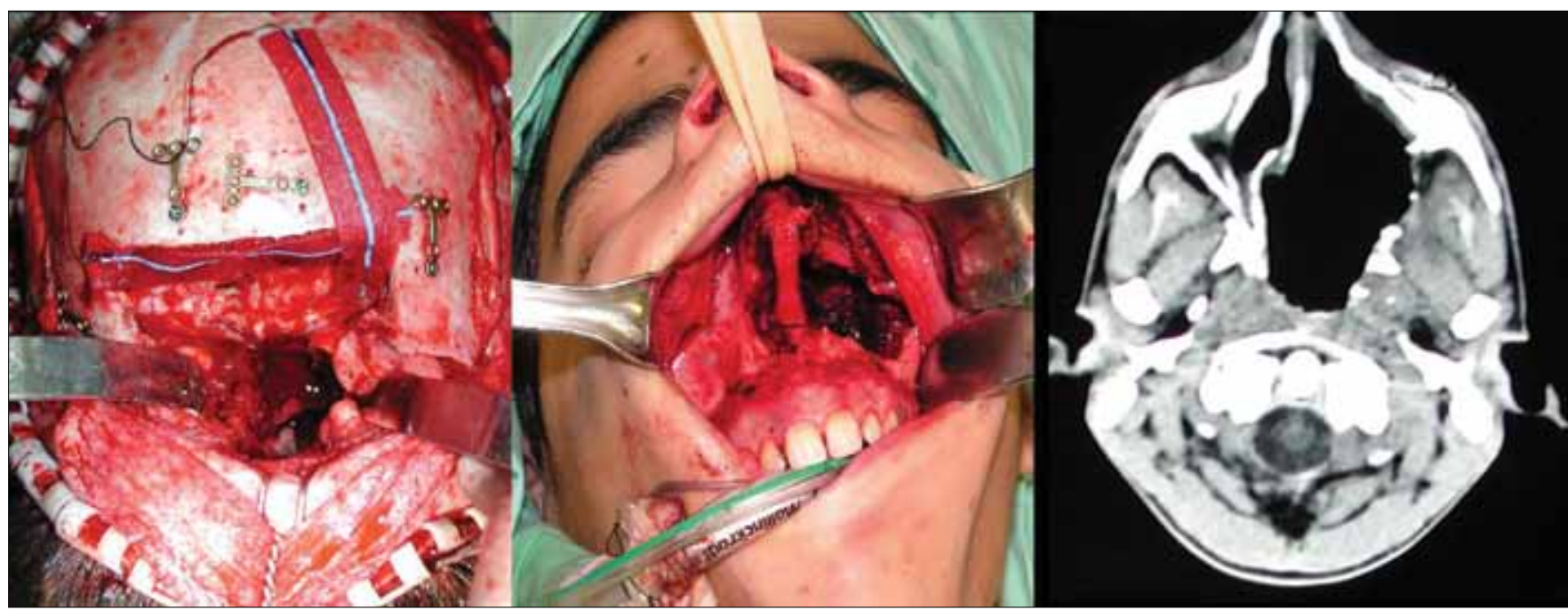

Figure 4. The subcranial-midfacial degloving (MFD) combined approach. Intraoperative view (middle and left) and CT scan post surgery (right) are shown

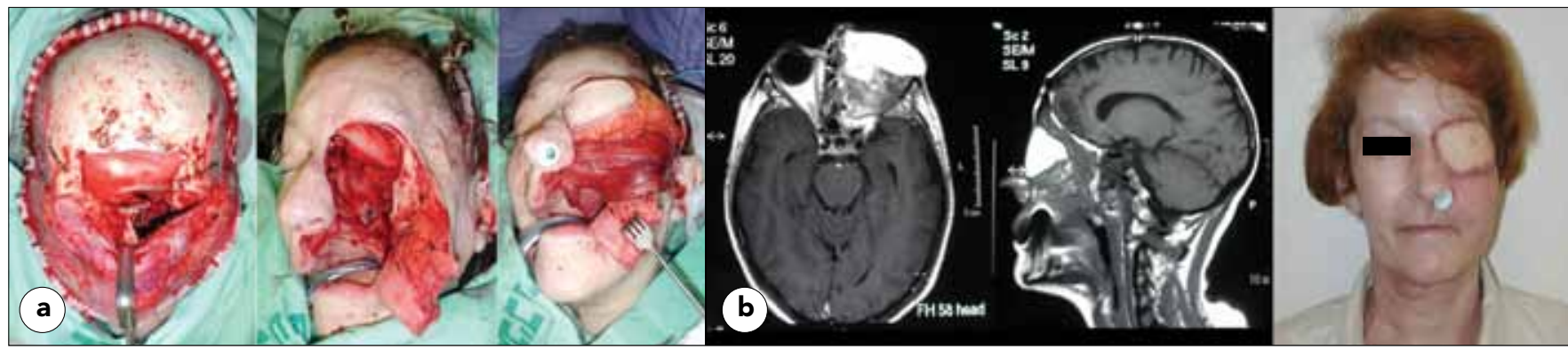

Figure 5. a) Intraoperative view for the subcranial-transorbital combined approach. b) The subcranial-transorbital combined approach. Postoperative CT scans (middle and left) and general appearance (right) are shown

The outcome of anterior skull base surgery has improved steadily over the years. In a systematic review by Dulgerov et al. (16), the overall disease-free survival in

The 1960s was $28 \pm 13 \%$, where as in the 1990 s it had improved to $51 \pm 14 \%$. To date, the best outcome studies of these tumours are from the International Collaborative Study, which published their results most recently in 2006 (1, 3, 17, 18). In this multicentre case series, in which our institution was included, data from 1307 patients were acquired retrospectively. Analysis of patient data from 1956-2000 showed a 5 -year overall survival of $54 \%$, including preoperative or adjuvant radiotherapy or chemotherapy, and an operative mortality of $4.3 \%$ was reported. Reduced 5 -year overall survival was associated with invasion of the orbital contents or brain, mucosal melanoma pathology, previous radiotherapy or chemotherapy, the presence of positive surgical margins, and the presence of comorbidities (1).

Although open surgical resection remains the gold standard for the removal of anterior skull base lesions, recent data indicate that endonasal endoscopic surgery is safe and is associated with acceptable outcomes in patients with benign and early malignant neoplasms (5). With advancements in imaging, diagnostic technology, diagnostic pathology, surgical technology and instrumentation, reconstructive techniques, and rehabilitation, it is expected that endoscopic approaches will evolve to round out the operative armamentarium.
Some issues such as disease extension over the orbit and into the orbit, extensive dural resection, and piecemeal excision are still to be resolved.

Ethics Committee Approval: N/A.

Informed Consent: N/A.

Peer-review: Externally peer-reviewed.

Author contributions: Concept - S.A-G., D.M.F.; Design - S.A-G., D.M.F.; Supervision - D.M.F.; Resource - S.A-G., D.M.F.; Materials S.A-G., D.M.F.; Data Collection\&/or Processing - S.A-G., D.M.F.; Analysis\&/or Interpretation - S.A-G., D.M.F.; Literature Search - S.A-G., D.M.F.; Writing - S.A-G., D.M.F.; Critical Reviews - D.M.F.

Conflict of Interest: No conflict of interest was declared by the authors.

Financial Disclosure: No financial disclosure was declared by the authors.

\section{References}

1. Patel SG, Singh B, Polluri A, Bridger PG, Cantu G, Cheesman AD, et al. Craniofacial surgery for malignant skull base tumors:report of an international collaborative study. Cancer 2003;98:1179-87. [CrossRef]

2. Ketchman AS, Wilkins RH, Vanburen JM, Smith RR. A combined intraacranial facial approach to the paranasal sinuses. Am J Surg 1963;106:698-703. [CrossRef]

3. Ganly I, Patel SG, Singh B, Kraus DH, Bridger PG, Cantu G, et al. Craniofacial resection for malignant paranasal sinus tumors:Report of an International Collaborative Study. Head Neck 2005;27:575-84. [CrossRef] 
4. Gil Z, Fliss DM. Tumours of the skull base and paranasal sinuses. 1st ed. Byword Books. 2011.

5. Mehta RP, Cueva RA, Brown JD, Fliss DM, Gil Z, Kassam AB, et al. What's new in skull base medicine and surgery? Skull Base Committee Report. Otolaryngol Head Neck Surg 2006;135:620-30. [CrossRef]

6. Gil Z, Fliss DM. Pericranial wrapping of the frontal bone after anterior skull base tumor resection. Plast Reconstr Surg 2005;116:395-8. [CrossRef]

7. Gil Z, Even-Sapir E, Margalit N, Fliss DM. Integrated PET/CT system for staging and surveillance of skull base tumors. Head Neck 2007;29:537-45. [CrossRef]

8. Margalit N, Wasserzug O, De-Row A, Abergel A, Fliss DM, Gil Z. Surgical treatment of juvenile nasopharyngeal angiofibroma with intracranial extension. Clinical article. J Neurosurg Pediatr 2009;4:113-7. [CrossRef]

9. Fliss DM, Zucker G, Amir A, Cohen JT, Gatot A. The combined subcranial-midfacial degloving approach. Operative Techniques in Otolaryngology-Head and Neck Surgery 2000;11:279-85. [CrossRef]

10. Mantravadi AV, Zender CA. Craniofacial approaches to the anterior skull base. Operative Techniques in Otolaryngology-Head and Neck Surgery 2010;21:181-7. [CrossRef]

11. Fliss DM, Zucker G, Cohen A, Amir A, Sagi A, Rosenberg L, et al. Early outcome and complications of the extended subcranial approach to the anterior skull base. The Laryngoscope 1999;109:153-60. [CrossRef]
12. Fliss DM, Abergel A, Cavel O, Margalit N, Gil Z. Combined subcranial approaches for excision of complex anterior skull base tumors. Arch Otolaryngol Head Neck Surg 2007;133:888-96. [CrossRef]

13. Fliss DM, Zucker G, Amir A, Gatot A, Cohen JT, Spektor S. The subcranial approach for anterior skull base tumors. Operative Techniques in Otolaryngology-Head and Neck Surgery 2000;11:238-53. [CrossRef]

14. Fliss DM, Gil Z, Spektor S, Leider-Trejo L, Abergel A, Khafif A, et al. Skull base reconstruction after anterior subcranial tumor resection. Neurosurg Focus 2002;12:e10. [CrossRef]

15. Zucker G, Nash M, Gatot A, Amir A, Fliss DM. The combined subcranial-pterional approach to the anterolateral skull base. Operative Techniques in Otolaryngology-Head and Neck Surgery 2000;11:286-93. [CrossRef]

16. Dulguerov $\mathrm{P}$, Jacobsen MS, Allal AS, Lehmann W, Calcaterra T. Nasal and paranasal sinus carcinoma:are we making progress? A series of 220 patients and a systematic review. Cancer 2001;92:3012-29. [CrossRef]

17. Ganly I, Patel SG, Singh B, Kraus DH, Bridger PG, Cantu G, et al. Complications of craniofacial resection for malignant tumors of the skull base:report of an International Collaborative Study. Head Neck 2005;27:445-51. [CrossRef]

18. Ganly I, Patel SG, Singh B, Kraus DH, Bridger PG, Cantu G, et al. Craniofacial resection for malignant melanoma of the skull base:report of an international collaborative study. Arch Otolaryngol Head Neck Surg 2006;132:73-8. [CrossRef] 